\title{
Conformational Flexibility Influences Structure-Function Relationships in Nucleic Acid N-Methyl Demethylases
}

\section{Sodiq O. Waheed, ${ }^{\text {a,\& }}$ Rajeev Ramanan, ${ }^{\text {a,\& }}$ Shobhit S. Chaturvedi, ${ }^{a}$ Jon Ainsley, ${ }^{\mathrm{b}}$ Martin Evison, ${ }^{b}$ Jennifer M. Ames, ${ }^{\text {c }}$ Christopher J. Schofield, ${ }^{d,}$ * Christo Z. Christov, ${ }^{\text {a,b }}{ }^{*}$ and Tatyana G. Karabencheva-Christova ${ }^{a, b^{*}}$}

a Department of Chemistry, Michigan Technological University, Houghton, Michigan 49931, United States.

${ }^{\mathrm{b}}$ Faculty of Health and Life Sciences, Northumbria University, Newcastle upon Tyne, NE1 8ST, United Kingdom.

${ }^{\mathrm{c}}$ Centre for Research in Biosciences, Faculty of Health and Applied Sciences, University of the West of England, Bristol, BS16 1QY, United Kingdom. Present address: Jenny Ames Consulting Ltd, Reading, RG6 5PY, United Kingdom.

d The Chemistry Research Laboratory, The Department of Chemistry, Mansfield Road, University of Oxford, Oxford, OX1 3TA, United Kingdom.

${ }^{\&}$ S.O.W., and R.R. made equal contributions to this article.

*Corresponding authors: C.J.S. (christopher.schofield@chem.ox.ac.uk), C.Z.C. (christov@mtu.edu), and T.G.K.C. (tatyanak@mtu.edu)

\begin{abstract}
N-Methylation of DNA/RNA bases can be regulatory or damaging and is linked to diseases including cancer and genetic disorders. Bacterial AlkB and human FTO are DNA/RNA demethylases belonging to the Fe(II) and 2-oxoglutarate oxygenase superfamily. Modelling studies reveal conformational dynamics influence structure-function relationships of AlkB and FTO, e.g. why 1-methyladenine is a better substrate for AlkB than 6-methyladenine. Simulations show that the flexibility of the double stranded DNA substrate in AlkB influences correlated motions, including between the core jelly-roll fold and an active site loop involved in substrate binding. The FTO N- and C-terminal domains move in respect to one another in a manner likely important for substrate binding. Substitutions, including clinically observed ones, influencing catalysis contribute to the network of correlated motions in AlkB and FTO. Overall, the calculations highlight the importance of the overall protein environment and its flexibility on the geometry of the reactant complexes.
\end{abstract}




\section{Introduction}

N-Methylation of DNA/RNA bases can be toxic causing mutagenesis or can be regulatory. ${ }^{1-4}$ 2Oxoglutarate (2OG) and Fe(II) dependent oxygenases play key roles in N-methyl demethylation of nucleic acids in organisms ranging from bacteria to humans (Fig. 1). ${ }^{4-7} 2 \mathrm{OG}$ oxygenases typically employ an active site containing a single Fe(II) ion ligated by one Asp and two His residues; ligation of water and 2OG (in a bidentate manner) completes a six coordinate (6C) octahedral geometry $^{8,9}$ (Fig. S1). AlkB is a bacterial repair enzyme with broad substrate specificity towards alkylated bases/exocyclic bridge-containing lesions. ${ }^{10,11}$ AlkB type enzymes are the likely precursors of eukaryotic $20 \mathrm{O}$ dependent nucleic acid oxygenases, some of which are linked to important biological processes and diseases. The Fat Mass and Obesity-Associated Protein (FTO) is an eukaryotic AlkB homolog which likely mainly demethylates N-methylated bases in RNA; mutations to the FTO gene in humans are linked to changes in body mass.$^{11-13}$

Crystal structures of $\mathrm{AlkB}^{10}$ and $\mathrm{FTO}^{5}$ reveal a distorted double stranded beta helix (DSBH, or 'jelly roll') catalytic core fold which is conserved in $2 \mathrm{OG}$ oxygenases. The nucleic acid oxygenase subfamily of $20 \mathrm{OG}$ oxygenases manifests characteristic elements, including an Arg-containing motif involved in 2OG C5 carboxylate binding. In addition to its core DSBH fold (aa. 91-214), AlkB contains an N-terminal extension (aa.13-44), a nucleotide recognition lid (aa. 45-90) and active site bordering loop (aa. 132-143). FTO has an N-terminal AlkB-like domain (aa. 32-326), a C-terminal domain (residues 327-498), and a loop involved in the recognition of single stranded substrates. AlkB and FTO recognize specific differently alkylated DNA/RNA bases ${ }^{1,2,11,14}$. Clinically observed mutations in FTO, e.g. R316Q, S319F, and R322Q correlate with pathological changes including growth retardation, developmental delay, and facial dysmorphism ${ }^{15-17}$. Other substitutions in AlkB (T51A, W69A, Y76A, D135A, R161A) and FTO (F114D, C392D) are reported to affect catalysis. ${ }^{5,18}$ Substitutions of the active site residues in AlkB and FTO have also been reported to result in changing of substrate selectivity. ${ }^{10}$

Studies on the modification of methyl groups on DNA and RNA are of interest from basic research and applied perspectives because of the importance of methylation in regulation and its ability to define particular types of tissues/cells. Methylation of DNA bases are part of normal gene expression and epigenetic processes, but abnormal DNA methylation patterns are characteristic of 
certain cancers and genetic disorders. Tissue and age-related methylation patterns are useful in forensic investigations of trace amounts of biological samples. ${ }^{19}$

Studies on nucleic acid oxygenases, including AlkB/FTO catalysis, ${ }^{20-24}$ indicate that they operate via the overall consensus mechanism for $2 \mathrm{OG}$ oxygenases (Scheme 1). The limited solution NMR studies on $20 \mathrm{O}$ oxygenases, including on AlkB and a prolyl hydroxylase, imply major conformational changes, especially for AlkB, during $\mathrm{Fe}(\mathrm{II})$, co-substrate and substrate binding/catalysis. ${ }^{25,26}$ However, there is not yet an understanding of how long-range conformational dynamics and correlated motions influence structure-function relationships. There is also incomplete knowledge regarding flexibility in the 2OG binding site; this is important with respect to understanding how $2 \mathrm{OG}$ co-substrate binding selectivity is achieved and in their inhibition, since most reported $2 \mathrm{OG}$ oxygenase inhibitors chelate Fe(II) and compete with 2OG. ${ }^{27}$ How conformational flexibility influences substrate selectivity, e.g. the experimental finding that 1-methyladenine (1MA is a better substrate than 6-methyladenine (6MA) for AlkB is also unclear.
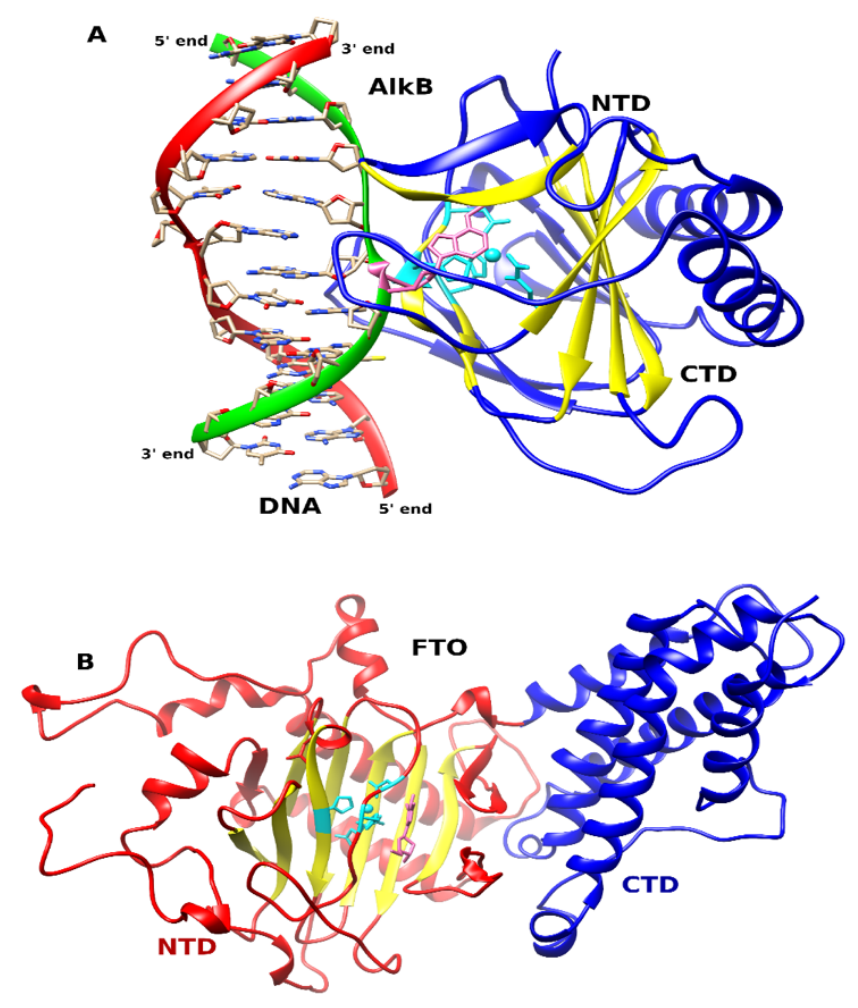

Fig. 1. Average structures of AlkB and FTO derived from MD simulations. Coloring: double stranded beta helix (DSBH) core fold (yellow), active site (cyan), and substrate (pink). 
We report investigations on this missing knowledge using long-range Molecular Dynamics (MD) simulations, free energy calculations, and Combined Quantum Mechanics and Molecular Mechanics (QM/MM) studies on enzyme-substrate complexes of representative nucleic acid oxygenases, i.e. AlkB and its larger and apparently more complex human homologue FTO. Related computational strategies have been successfully implemented for investigating structure-function relationships of various other enzymes. ${ }^{28-33}$ The results show conformational dynamics exercise distinct effects in AlkB and FTO and are important in determining substrate selectivity. Specific residues in AlkB/FTO play important roles in correlated motions/flexibility and are important in maintaining the productive geometry of reactant complexes.

A
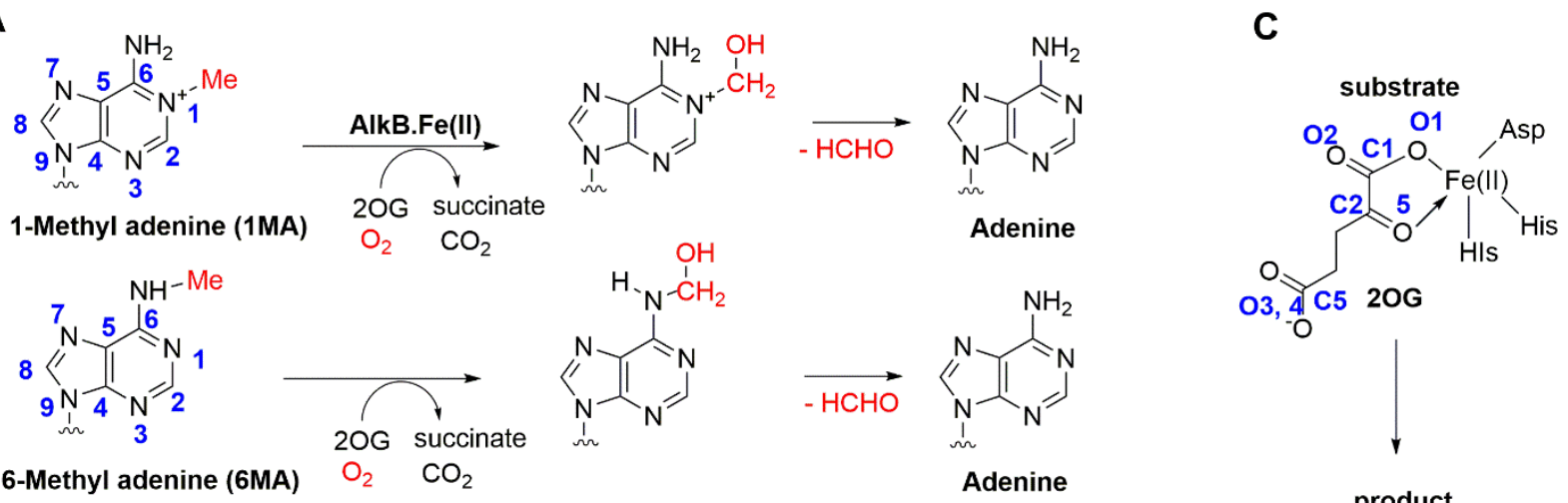

6-Methyl adenine (6MA)

B<smiles>Cc1cn(C)c(=O)n(C)c1=O</smiles>

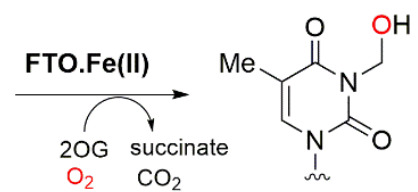

3-Methyl thymine (3-meT)

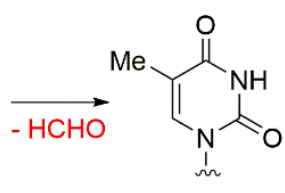

Thymine

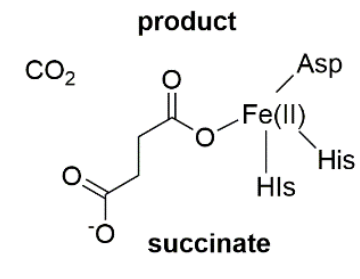

Scheme 1: Reactions catalysed by AlkB (A) and FTO (B). Demethylation proceeds via hydroxylation, which is mediated via an $\mathrm{Fe}=\mathrm{O}$ (IV) intermediate, to form a hemiaminal which fragments to give formaldehyde and the product. (C) Both the AlkB and FTO reactions are coupled to the conversion of 2-oxoglutarate and $\mathrm{O}_{2}$ to succinate and $\mathrm{CO}_{2}$.

\section{Computational Methods}

2.1. System Preparation: An X-ray crystal structure of FTO (PDB ID code: 4IDZ ${ }^{3}$ ) was used for building the FTO model used in the computational studies. Missing residues from loop regions 
were added using Modeller. ${ }^{34}$ A crystal structure of the AlkB (PDB: 4NID ${ }^{10}$ in complex with Mn(II), 2-oxoglutarate (2OG) and double stranded DNA containing N6-methyladenine (6MA)) was used in computational studies. The $\mathrm{Mn}(\mathrm{II})$ was replaced with $\mathrm{Fe}(\mathrm{II})$. AlkB was also modelled with the nucleoside only (6MA (nsd)) instead of double stranded DNA to compare with the FTO single base simulations and to investigate how the DNA affects the conformational dynamics of AlkB. A wildtype structure of AlkB (PDB: $3 \mathrm{BIE}^{35}$ ) with substrate N1-Methyladenine (m1A) was also used in simulation studies. The parameters for the substrate were developed using Antechamber. The likely protonation states of ionisable sidechains of the proteins were assessed using the $\mathrm{H}++$ server. $^{36}$ Histidine residues coordinating with the metal centre were assigned protonation states based on visual inspection of their local environment. The Amber parameters for the active site containing $\mathrm{Fe}(\mathrm{II})$ high spin $\mathrm{S}=2, \mathrm{M}=5$, ground state $8,9,14,22$ and the coordinating ligands (2OG, with bidentate ligation), histidine, and aspartic acid (both via monodentate ligation) were prepared using the Metal Centre Parameter Builder (MCPB) using MCPB.py v1.0 Beta2 ${ }^{37}$ for a 5-coordinate $(5 \mathrm{C})$ distorted square pyramidal active site.

2.2. MD Simulations: Molecular dynamics simulations were performed using the GPU version ${ }^{38}$ of PMEMD engine integrated with Amber $14 .{ }^{39}$ The FF14SB ${ }^{40}$ force field was used in all the simulations and the Leap module was used to add missing hydrogen atoms and counter ions for neutralisation of the protein system. All the systems were immersed into a truncated octahedral box with TIP3P water molecules, ${ }^{41}$ such that no protein atom was within $10 \AA$ of any box edge. Periodic boundary conditions were employed in all the simulations. Long-range electrostatic interactions were calculated using the Particle Mesh Ewald (PME) method ${ }^{42}$ with a direct space and vdW cut-off of $8 \AA$. The various systems were subjected to energy minimization using first steepest descent (5000 steps) followed by conjugate gradient (5000 steps) to eliminate clashes. Solute molecules were restrained using a restrained potential of $100 \mathrm{kcal} \mathrm{mol}^{-1} \AA^{2}$; only solvent and ions were allowed to minimize. This was followed by full minimization of the entire system with both steepest descent (5000 steps) and conjugate gradient (5000 steps) treatments to relax the system prior to productive simulation. The systems were then subjected to controlled heating from 0 to $300 \mathrm{~K}$ at constant volume using the Langevin thermostat ${ }^{43}$ with a collision frequency of 1 $\mathrm{ps}^{-1}$ using a canonical ensemble (NVT) MD simulation for $400 \mathrm{ps}$. The solute molecules were restrained using a harmonic potential of $10 \mathrm{kcal} \mathrm{mol}^{-1} \AA^{2}$ during the heating process. The SHAKE algorithm ${ }^{44}$ was used to constrain bonds involving hydrogen. This was followed by equilibration 
at $300 \mathrm{~K}$ in an isothermal-isobaric ensemble (NPT) for $1 \mathrm{~ns}$ without restraints on solute molecules. The pressure was maintained at 1 bar using the Berendsen barostat. ${ }^{45}$ A productive MD run with explicit solvent for continuous $1 \mu$ s was performed in an NPT ensemble with a target pressure set at $1 \mathrm{bar}$ and constant pressure coupling of $2 \mathrm{ps}$. The frames from the productive run were saved every 10 ps. Trajectories were analysed using CPPTRAJ, ${ }^{46}$ VMD,,${ }^{47}$ UCSF Chimera, ${ }^{48}$ and $\mathrm{R}$ $\left(\mathrm{Bio3}^{49}\right)$.

2.3. QM/MM calculations: Snapshots for the $\mathrm{QM} / \mathrm{MM}$ calculations were obtained from the $\mathrm{MD}$ simulations on all the systems. These snapshots were first subjected to energy minimization for 10,000 steps by using both steepest descend (5000) and conjugate gradient (5000) algorithms in Amber14. Active site residues were restrained with a restrained potential of $100 \mathrm{kcal} \mathrm{mol}^{-1} \AA^{2}$ in the energy minimization, to maintain the geometry of the active site. The energy minimized snapshots of all the enzymes were prepared using the Schlegel's toolkit $\mathrm{TAO}^{50}$ for $\mathrm{ONIOM}^{51-55}$ calculation in Gaussian09. ${ }^{56}$ Residues within $20 \AA$ of Fe(II), including water molecules, were allowed to move freely during geometry optimization; the rest of the system was frozen during geometry optimization in ONIOM. Hydrogen atoms were used as the link atoms to saturate the dangling bond in the QM/MM calculation. ${ }^{57}$ The electrostatic embedding scheme was used in the geometry optimization however, we also used the mechanical embedding scheme for some snapshots.

2.4. Molecular Mechanics/Generalized Born Surface Area (MM/GBSA): The binding free energy calculations with AlkB were performed using the Molecular Mechanics/Generalized Born Surface Area (MM/GBSA) approach. ${ }^{58-60}$ More details of computational methods are provided in the Supporting Information.

\section{Results and Discussion}

MD simulations of the complex of AlkB with Fe(II), $2 \mathrm{OG}$ and DNA substrate containing 6-methyl adenine (AlkB-6MA(DNA) imply the Fe(II) centre and its ligands (His131, His187, and Asp133) are conformationally stable, as are second sphere interactions of (i) the two ligating histidines with C129 and F185, and (ii) the interaction of D133 with W178 (Fig. S2). However, interactions between the non-coordinating carboxyl oxygen of the monodentate ligating D133 with R210 are strengthened compared to the crystal structure, in agreement with NMR studies showing the 
presence of a hydrogen bond stabilising monodentate coordination of the Fe(II) ligating carboxylate in some $2 \mathrm{OG}$ oxygenases ${ }^{25}$ (Fig. S2).

To explore the effects of the flexibility of the DNA substrate on the enzyme-substrate complex, we performed simulations with AlkB complexed with Fe(II), 2OG and 6MA nucleoside (AlkB$6 \mathrm{MA}$ (nsd)). The results reveal that the average distance between the substrate methyl group and $\mathrm{Fe}$ (II) is not substantially different in AlkB-6MA (DNA) and AlkB-6MA (nsd) (Fig. 2) indicating that this interaction is not influenced by the bulk DNA, at least once a substrate is bound.

1-Methyl adenine (1MA) is reported to be a better AlkB substrate than 6MA. ${ }^{10,11,14}$ To investigate the atomistic reasons for this, we simulated the AlkB, Fe(II), 2OG with the 1MA nucleoside (AlkB1MA (nsd)). The simulations show that the distance between the methyl group of 1MA and Fe(II) is stable during the simulation and is lower compared to the analogous distances in AlkB6MA(nsd) and AlkB-6MA(DNA) (Fig. 2). An apparently key hydrogen bond between the N6 of $1 \mathrm{MA}$ and D135, as observed in the crystal structure, is stable with 1MA in contrast to 6MA in AlkB-6MA (nsd) and AlkB-6MA (DNA), consistent with 1MA being a better substrate for AlkB than 6MA (Fig. 3).

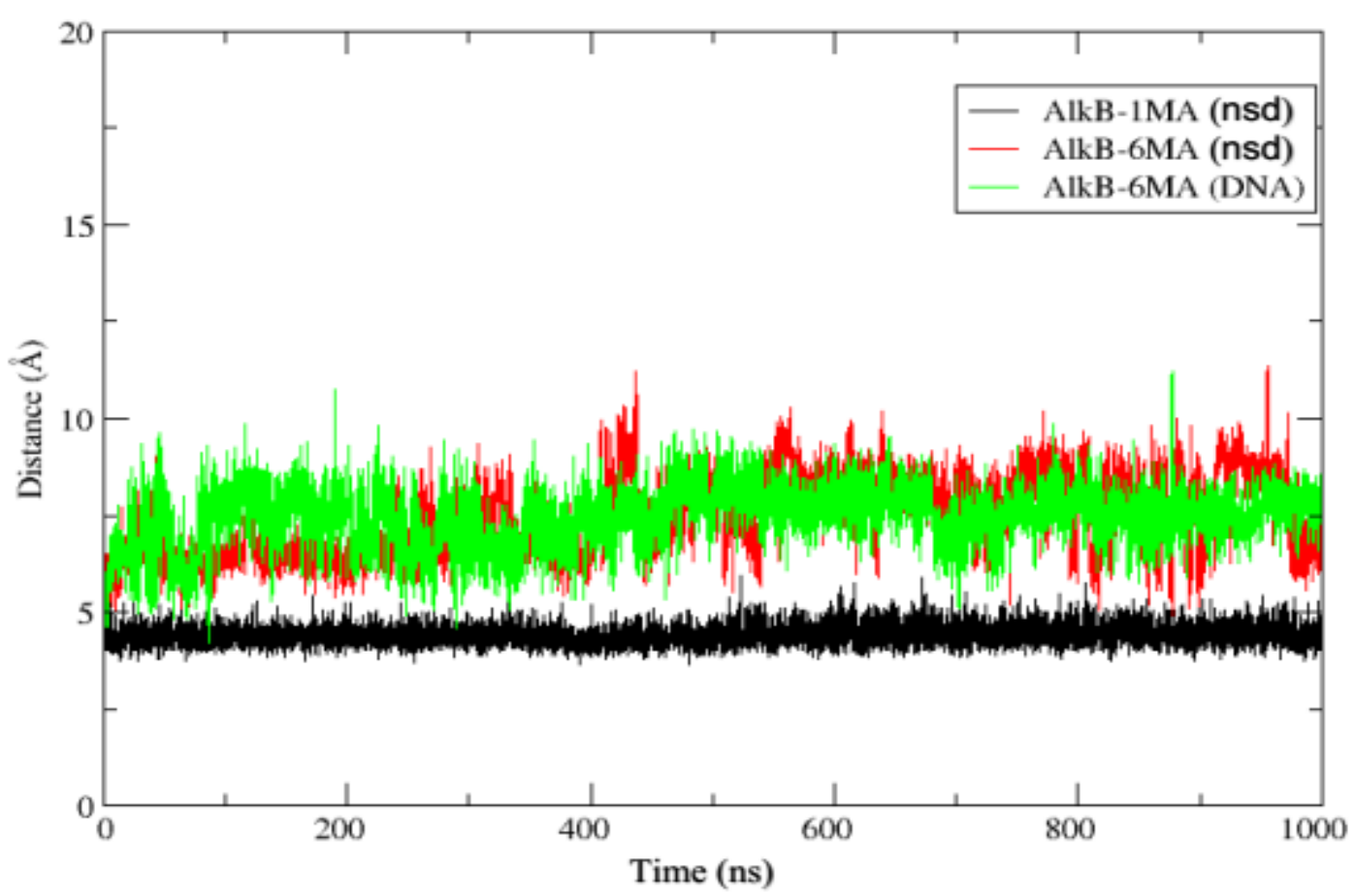

Fig. 2. The distance between Fe(II) and the carbon atom of the substrate methyl group in AlkB1MA (nsd), AlkB-6MA (nsd), and AlkB-6MA (DNA) during the MD simulations. The results 
simply the overall binding mode of 6MA at the active site is not strongly affected depending if it is in a nucleic acid sequence or not. $1 \mathrm{MA}$ is apparently better positioned for demethylation than $6 \mathrm{MA}$ during the entire simulations.

The adenine ring in AlkB-1MA (nsd) is bound/orientated by stable interactions with the H131, W69, and Y78 side chains. With 6MA, stacking interactions of the adenine ring of AlkB-6MA (DNA) and AlkB-6MA (nsd) with W69 and H131 manifest more flexibility than with 1MA. The stability of the interactions in $1 \mathrm{MA}$ likely reflects its stronger binding in the active site of the AlkB compared with 6MA. To calculate the binding affinities of 1MA and 6MA, we performed free energy calculations; the MMGBSA method gave relative free energies of binding of -4.8 for AlkB$1 \mathrm{MA}$ ) and $-2.0 \mathrm{kcal} / \mathrm{mol}$ for AlkB-6MA) (Table S1), again consistent with the experimental observations $^{10}$.

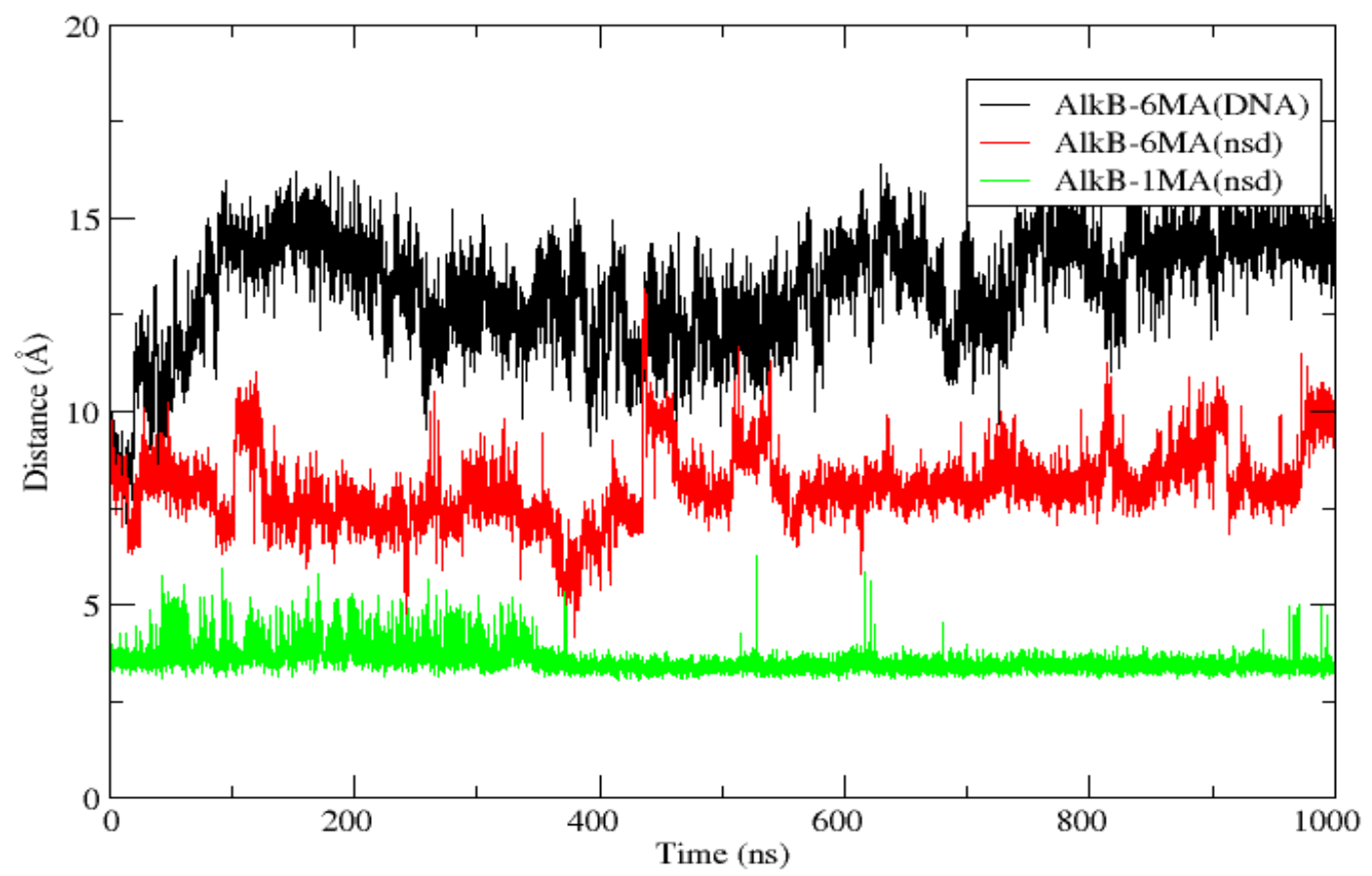

Fig. 3. The hydrogen bond interactions of the N6 of the substrates (1MA(nsd), $6 \mathrm{MA}(\mathrm{nsd})$ and 6MA(DNA)) with D135 in AlkB. Distances were measured between the centres of masses of the substrates and the carboxyl group of the ligating aspartate (D135). 
In FTO, Fe(II), $2 \mathrm{OG}$ and the N-methyl group of the 3-methyl thymidine (3-meT) substrate make stable interactions (Fig. S3). The Y108 and H132 side chains are positioned to stabilize the 3-meT substrate ring via $\pi-\pi$ stacking interactions (Fig. S3); the deoxyribose ring of 3-meT is stabilized by the L109 side chain, as proposed from crystallographic analyses ${ }^{12}$ (Fig. S3). A hydrogen bond between E234 and O4 of 3-meT and a hydrophobic cluster formed by L203, Y106, and Y108 involved in binding the 3-meT methyl group are also stable (Fig. S3). R96 is involved in binding both the non-ligating $\mathrm{C} 1$ carboxylate oxygen $(\mathrm{O} 1)$ of $2 \mathrm{OG}$ and 3-meT, the latter via hydrogen bonds with $\mathrm{O} 2$ of 3-meT. The key role of R96 is confirmed by inhibition ${ }^{61,62}$ and mutagenesis studies, including by showing the R96H variant manifests reduced activity. ${ }^{7}$

In AlkB, R204 makes a stable electrostatic interaction with the non-coordinating $2 \mathrm{OG}$ C5 carboxylate with an average distance of $4.0 \AA$ (Fig. S4). The C5 carboxylate of $2 \mathrm{OG}$ is further stabilised by hydrogen bonds with the side chains of Y122, N120, and N206, as observed crystallographically. ${ }^{10}$ The R210 side chain is positioned to hydrogen bond with the D133 carboxylate in AlkB-1MA (nsd), in contrast to AlkB-6MA (DNA) and AlkB-6MA (nsd) where limited interactions are observed (Fig. S5). The hydrogen bond analysis supports the presence of hydrogen bonds interaction between the non-coordinating 2OG carboxylate oxygen atoms $(\mathrm{O} 3$, O4) and R204 (94\%), Y122 (93\%), while potential hydrogen bonds between 2 OG and N206 (1\%) and N120 (1\%) are unstable indicating some flexibility in the 2OG binding site (Fig. S6).

In FTO, the 2OG C5 carboxylate is bound by interactions with R316, Y295 and S318; R204, Y122, N120 and N206 serve the same purpose in AlkB (Fig. S6). Hydrogen bonds between the noncoordinating oxygen $(\mathrm{O} 1)$ of $\mathrm{C} 1$ of $2 \mathrm{OG}$ in FTO with the side chains of R96 and N205 which are observed in the crystal structure show limited stability (30\% and $36 \%$, respectively) during the MD trajectories. In FTO, hydrogen bonds stabilising the non-coordinating C5 carboxylate of $2 \mathrm{OG}$ (O3 and O4) group are found to be more stable - R316 (98\%) S318 (97\%) and Y295 (77\%). Overall, the MD simulations indicate some flexibility in the 2OG binding sites in both AlkB (in agreement with NMR data ${ }^{25}$ ) and FTO which might be important for the design of enzyme-specific $2 \mathrm{OG}$ analogues. 
In the AlkB-6MA(DNA) complex, the DNA has an average RMSD value of $4.0 \AA$ (Fig. S7), by contrast to $1.4 \AA$ for the protein alone. This result indicates that DNA contributes to the relatively high flexibility of the complex in agreement with other simulations of AlkB with dsDNA. ${ }^{33}$ NMR studies also reveal the importance of the DNA conformation in dynamics of the overall complex. ${ }^{25}$ The distance between the centre of mass of protein and DNA was $28.2 \AA$, larger than the same distance observed in the crystal structure (24.0 $\AA$ ) of the AlkB-6MA(DNA) complex (Fig. S8). This observation indicates a more open structure of the AlkB-6MA(DNA) than the crystallographic study indicates, likely reflecting crystal packing effects. AlkB-6MA (nsd) and AlkB-1MA (nsd) showed equilibration with an average RMSD value of $1.4 \AA$ (Fig. S9), further supporting the crystallographic observations that AlkB-6MA (nsd) and AlkB-1MA (nsd) manifest near identical structures apart from the active site loop. ${ }^{10}$ The active site loop of AlkB-6MA (nsd) and AlkB-6MA (DNA) manifests a conformational change around 200-250ns and greater structural fluctuations in contrast to the AlkB-1MA (nsd) which could contribute to the weaker binding of 6MA (Fig. S10). The DSBH core shows low structural fluctuations which probably helps to maintain a stable environment for the $\mathrm{Fe}(\mathrm{II})$ centre and co-substrates/intermediates, possibly to help prevent self-oxidation. ${ }^{63}$

Principal Component Analysis (PCA) suggests that the AlkB-6MA(DNA) complex mainly manifests motion in its the active site loop region and $\mathrm{N}$ - and $\mathrm{C}$-terminal regions; the methylated DNA base showed limited flexibility (Fig. 4A and S11). However, AlkB-1MA (nsd) showed more limited motion of the active site loop (Fig. S12 and S13) which would contribute to the stronger binding of 1MA in AlkB-1MA (nsd) than the 6MA in AlkB-6MA (nsd).

Dynamic Cross Correlation Analysis (DCCA) was performed to investigate collective, correlated motions in AlkB and FTO. In AlkB-6MA (DNA) residues of the active site loop (133-139; $\beta 6-\beta 7)$ show correlation with residues 187-195 in the DSBH (loop linking $\beta 11$ and $\beta 12$ ) (Fig. 5). Residues 152-160 in the loop near the DNA show correlation towards residues 187-195 of the DSBH. However, there is reduction in correlated motion in the AlkB-6MA (nsd) and AlkB-1MA (nsd) systems compared to AlkB-6MA (DNA) complex (Fig. 5). These results thus support the proposal of important contributions by DNA dynamics to collective motions of the enzyme-substrate complex.

Experimental studies show that the T51A, W69A, Y76A, D135A, and R161A substitutions adversely affect AlkB catalysis ${ }^{18}$ (Fig. S14). From the DCCA (Fig. 5), D135 and R161 show 
positive correlations with the coordinating aspartic acid (D133) and histidines (H131 and H187) respectively. Thus, the D135A and R161A substitutions will likely affect the functioning of the catalytic domain. T51, W69 and Y76 also show positive correlations with the nucleotide recognition lid residues, which is involved in substrate binding ${ }^{10}$, thus the T51A, W69A and Y76A substitutions likely affect substrate binding.

The FTO NTD manifests larger structural deviations compared to the CTD, due to the larger number of loop regions in NTD. The linker connecting NTD to the CTD shows lower RMSDs indicating a stable connection between the two domains. The PCA shows that the main motion involves residues from the L1 loop in the NTD region (Fig. 4B and S15) and a movement of the CTD towards the NTD domain. DCCA (Fig. 5) shows that residues 90-115 of NTD have a positive correlation with CTD residues 380 to 390 from $\alpha 8$ helix. These motions might contribute to substrate binding by FTO and may be used for modulation of enzyme activity.
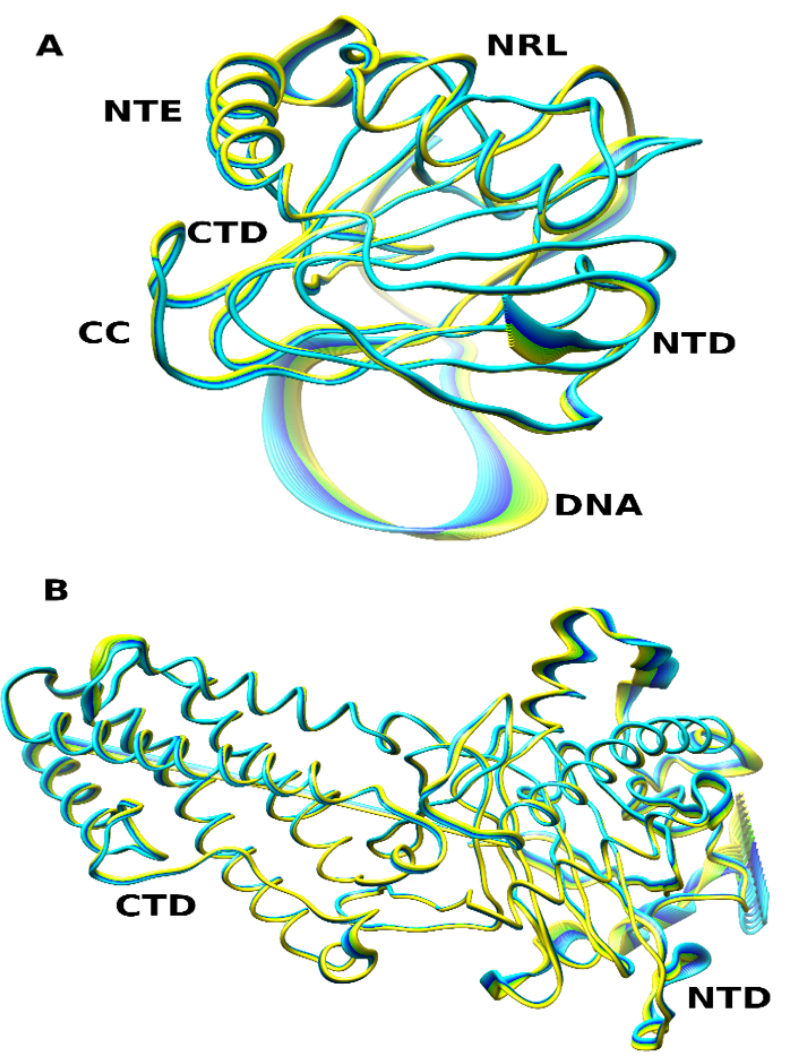

Fig. 4. Principal Component Analysis for AlkB-6MA (DNA) (A) and FTO (B). NTE: N-terminal extension; NRL: nucleotide-recognition lid; CC is the catalytic core while NTD and CTD are the $\mathrm{N}$-terminal domain and $\mathrm{C}$-terminal domain, respectively. Yellow to blue represents the main direction of motion of proteins residues. 

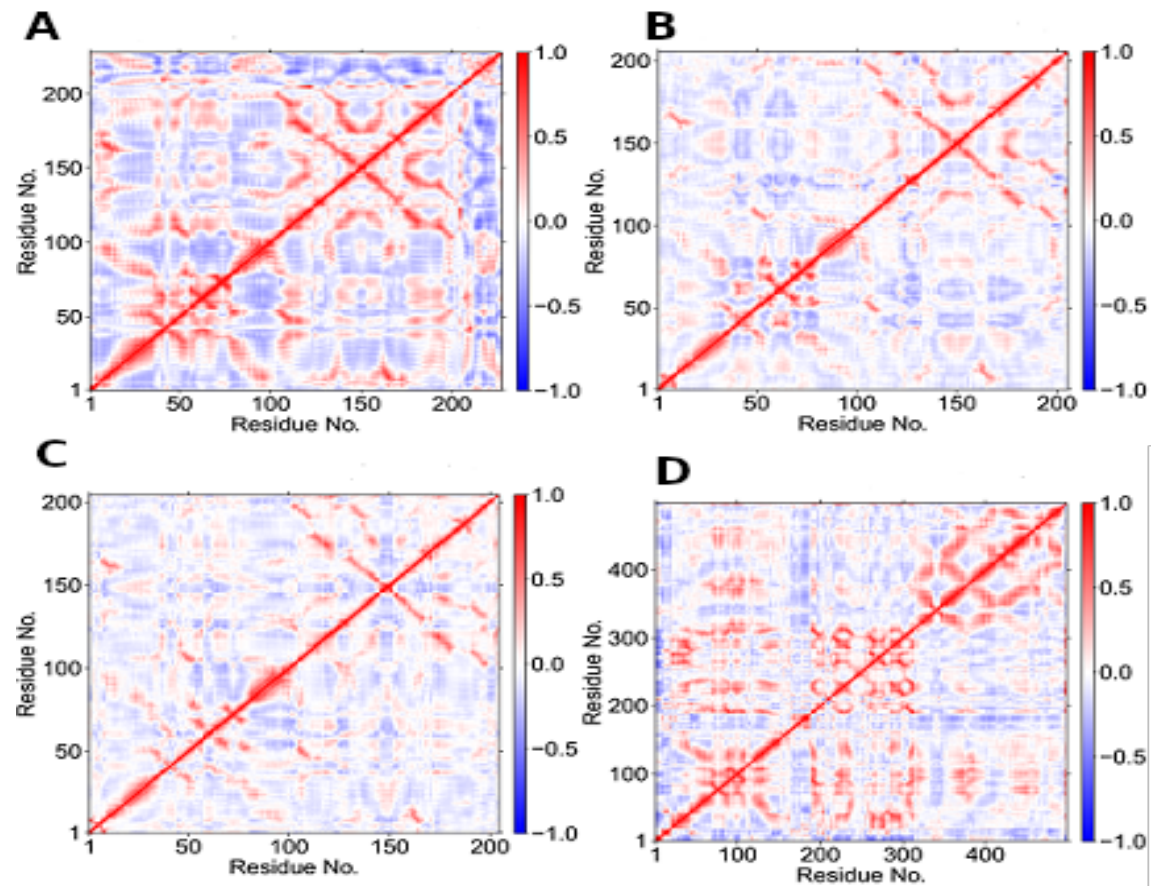

Fig. 5. Dynamic Cross Correlation for AlkB-6MA (DNA) (A), AlkB-6MA (nsd) (B), AlkB-1MA (nsd) (C) and FTO (D). Residue numbers in (A): 1-203 (protein), 204 (2OG), 205 (Fe), 206-229 (DNA), and 211 (substrate). (B) 1-203 (protein), 204 (2OG), 205 (Fe) and 206 (substrate). (C) 1202 (protein), 203 (2OG), 204 (Fe) and 205 (substrate). (D) 1-495 (protein), 496 (2OG), 497 (Fe), and 498 (substrate).

Mutations of F114 or C392 (F114D, C392D) (Fig. S16), which are involved in this correlated motion, might be involved in the disruption of inter-domain interactions and thus to lead to reduction in FTO activity, as observed experimentally ${ }^{5}$. Notably, the clinically observed mutations S319F and R322Q ${ }^{15-17}$ (Fig. S16) have positive correlations with the Fe(II) coordinating residues (H231, D233, H307), Fe, 2OG and the substrate (m3T), while R316Q has positive correlation with all the aforementioned, except the coordinating D233. These mutations are thus predicted to alter the correlated dynamics within the active site, including with respect to the binding of the substrate, in a manner possibly related to the reported pathologies. ${ }^{15-17}$

To further investigate the effects of the protein environment and flexibility on the geometric and electronic structure of the reactant complexes, $\mathrm{QM} / \mathrm{MM}$ geometry optimizations of the enzymesubstrate complexes of AlkB and FTO were performed. The Fe(II) coordination geometry of AlkB was maintained during QM/MM optimizations (Fig. 6 and Table S2). The second sphere interactions of the AlkB enzyme analysed from QM/MM snapshots are presented in Table S3. For 
example, the MD simulations and QM/MM optimizations of various snapshots manifest a consistent distance of $\sim 3.9 \AA$ between the side chain of R204 and the 2 OG C5 carboxylate. These $\mathrm{QM} / \mathrm{MM}$ results are consistent with the MD and crystallographic studies with AlkB.

Superposition of the structures of AlkB-1MA (nsd) and AlkB-6MA (nsd) optimized using QM/MM imply that the complex with $1 \mathrm{MA}$ is more productive compared to that of AlkB-6MA (nsd), based on the closer positioning of the methyl group of 1MA to the non-heme Fe (II) (Fig. 6), consistent with the experimental observations ${ }^{10}$ and the MD simulations. Superposition of the QM/MM optimized structures of the AlkB-6MA (nsd) and AlkB-6MA (DNA) complexes (using snapshots from the MD) shows overall consistency with the trends observed in MD; however, small differences are apparent (Fig. S17 and S18) indicating the importance of performing QM/MM minimizations.

With FTO, the Fe(II) coordination geometry and active site interactions also remained stable during QM/MM optimizations of MD snapshots (Fig. 7). Thus, the QM/MM optimizations manifested a consistent distance of $\sim 4.0 \AA$ between the R316 side chain and the $2 \mathrm{OG}$ C5 carboxylate $2 \mathrm{OG}$, validating the role of this electrostatic interaction.

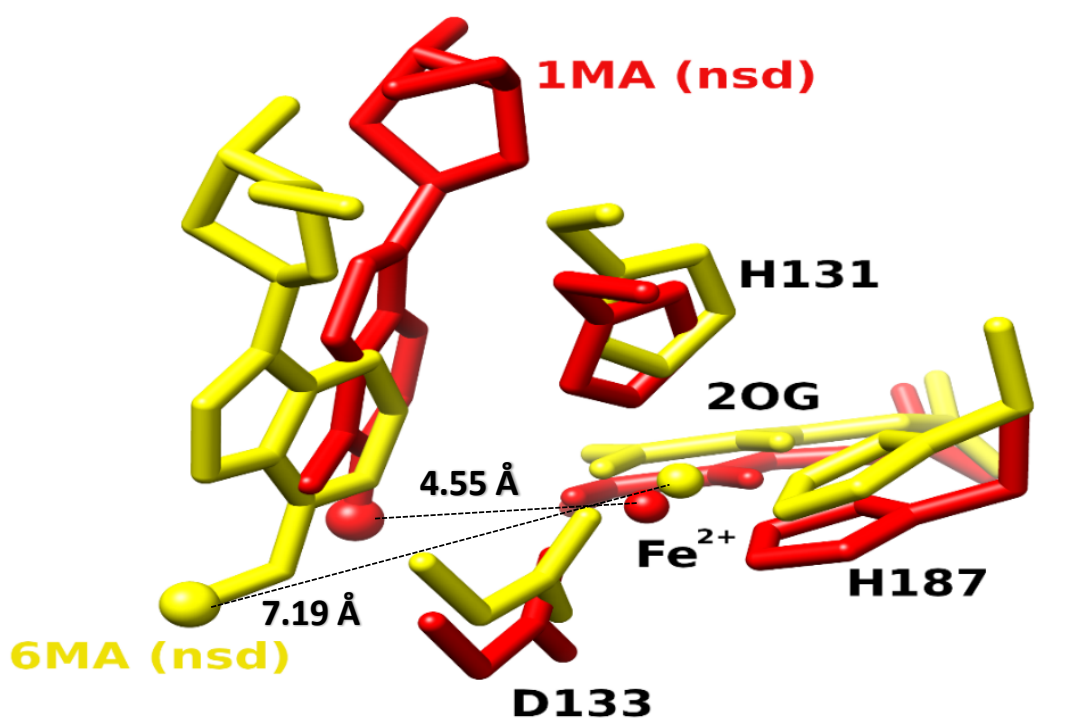

Fig. 6. Overlaid QM/MM optimized structures of the AlkB-1MA and AlkB-6MA complexes (with $\mathrm{Fe}(\mathrm{II})$ and 2OG) showing the active site. The methyl groups are shown as larger spheres. 

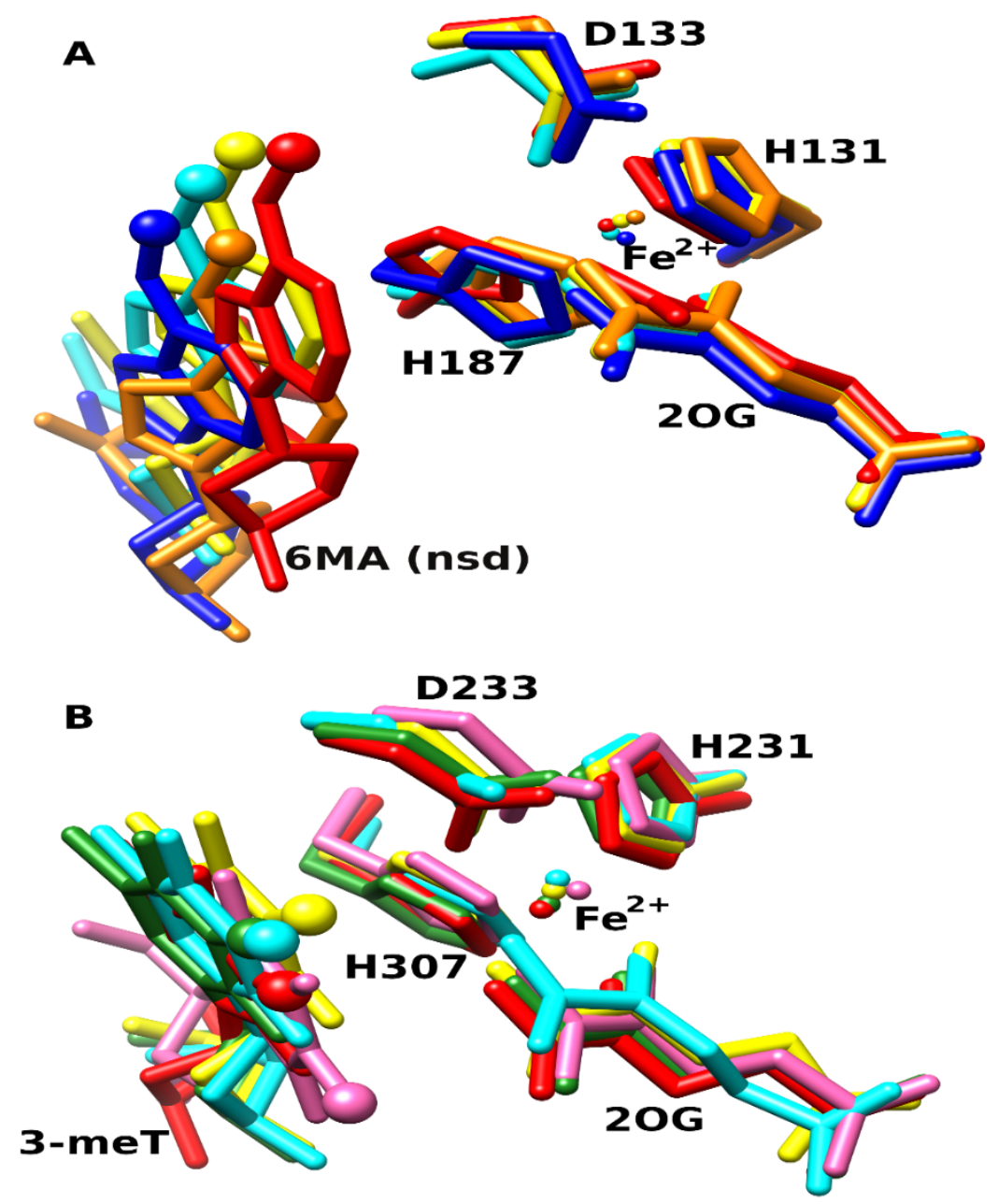

Fig. 7. Overlaid multiple QM/MM optimized snapshots of AlkB-6MA at: (A) at 200ns (orange), 300ns (yellow), 400ns (cyan), 500ns (blue), and FTO (B) at 200ns (red), 300ns (green), 700ns (cyan), 800ns (pink). The red and yellow structures in A and B represent views from QM/MM minimized structures of AlkB and FTO, respectively. The overlay was done using Chimera. The methyl group is shown as the larger sphere.

Comparison of the structures of the QM/MM optimized MD snapshots of AlkB-6MA (DNA) (Fig. 7A, Table S2) and FTO-3MT (nsd) (Fig. 7B and Table S4) also indicate that in both enzymes there are fine structural differences in the reactant complexes due to conformational fluctuations (in respect to $\mathrm{QM} / \mathrm{MM}$ minimized initial crystal structures), thus supporting the importance of accounting for the protein conformational dynamics in reaction mechanism calculations. 


\section{Conclusions}

The results of our experimentally-based multilevel computational studies on two homologous Nmethyl nucleic acid demethylases, bacterial AlkB and human FTO, complement reported biophysical (crystallographic and NMR) and kinetic studies of these enzymes, ${ }^{10,13,25}$ and provide new insights into the effects of the conformational dynamics, collective motions, and electronic structural features, which are difficult to define experimentally. The results show that the flexibility of the dsDNA substrate has a substantial effect on the dynamics and correlated motions in AlkBsubstrate complexes, including motions between the core jelly-roll motif and an active site loop involved in substrate binding. The results inform on how key residues in AlkB-and FTO influence activity via modulating correlated motions. The computations also inform on the underlying reasons why $1 \mathrm{MA}$ is a better substrate for AlkB than 6MA; future analogous studies on the selectivities of other nucleic acid oxygenases acting on both DNA and various forms of RNA are thus of interest. Most reported 2OG oxygenase inhibitors, including compounds in clinical applications, bind at the active site and ligate $\mathrm{Fe}(\mathrm{II})$, with consequent issues with respect to achieving selectivity. ${ }^{64}$ Our computational studies with FTO reveal its CTD and NTD domains move in respect to one another in a manner which likely important for substrate binding, an observation that suggests new opportunities for selective inhibition of some $2 \mathrm{OG}$ oxygenases via binding away from the active site.

\section{Conflicts of Interest}

There are no conflicts to declare.

\section{Acknowledgements}

CZC and TGKC acknowledge start-up grants from Michigan Technological University. CJS thanks the Wellcome Trust and Cancer Research UK for funding. The authors also thank Prof. Javier Luque from University of Barcelona for helpful discussions.

\section{References}

1. C. Loenarz, W. Ge, M.L. Coleman, N.R. Rose, C.D. Cooper, R.J. Klose, P.J. Ratcliffe and C.J. Schofield, Hum. Mol. Genet. 2010, 19, 217-222. 
2. C.J. Schofield and Z. Zhang, Curr. Opin. Struct. Biol.1999, 9, 722-731.

3. W. Aik, M. Demetriades, M.K.K. Hamdan, E.A.L. Bagg, K.K. Yeoh, C. Lejeune, Z. Zhang, M.A. McDonough and C.J. Schofield, J. Med. Chem. 2013, 56, 3680-3688.

4. I.J. Clifton, M.A. McDonough, D. Ehrismann, N.J. Kershaw, N. Gravatino and C.J. Schofield, J. Inorg. Biochem., 2006, 100, 644-669.

5. Z. Han, T. Niu, J. Chang, X. Lei, M. Zhao, Q. Wang, W. Cheng, J. Wang, Y. Feng, and J. Chai, Nature 2010, 464, 1205-1209.

6. Y, Fu, G. Jia, X. Pang, R.N. Wang, X. Wang, C.J. Li, S. Smemo, Q. Dai, K.A. Bailey, M.A. Nobrega, K.L. Han, Q. Cui and C. He, Nat. commun. 2013, 4, 1798.

7. D, Meyre, K. Proulx, H. Kawagoe-Takaki, V. Vatin, R. Gutierrez-Aguilar, D. Lyon, M. Ma, H. Choquet, F. Horber, W. Van Hul, L. Van Gaal, B. Balkau, S. Visvikis-Siest, F. Pattou, I.S. Faroogi, V. Saudek, S. O’Rahilly, P, Froguel, B. Sedgwick and G.S. Yeo, Diabetes 2010, 59, 311-318.

8. E.I. Solomon, T.C. Brunold, M.I. Davis, J.N. Kemsley, S. Lee, N. Lehnert, F. Neese, A.J Skulan, Y. Yang and J. Zhou, Chem. Rev. 2000, 100, 235-350.

9. R.H. Holm and E.I. Solomon, Chem. Rev. 2014, 114, 4039-4040.

10. C. Zhu and C. Yi, Angew. Chem. Int. Ed. 2014, 53, 3659-3662.

11. B.I. Fedeles, V. Singh, J.C. Delaney, D. Li and J.M. Essigmann, J. Biol. Chem. 2015, 290, 20734-20742.

12. G. Zheng and C. He, in 2-Oxoglutarate-Dependent Oxygenases, ed. C.J. Schofield and R.P. Hausinger, Royal Society of Chemistry, Cambridge, 2015, series No. 3, ch. 9, pp. 263-274.

13. T. Gerken, C.A. Girard, Y.L. Tung, C.J. Webby, V. Saudek, K.S. Hewitson, G.S.H. Yeo, M.A. McDonough, S. Cunliffe, L.A. McNeil, J. Galvanovskis, P. Rorsman, P. Robins, X. Prieur, A.P. Coll, M. Ma, Z. Jovanovic, I.S. Farooqi, B. Sedgwick, I. Barroso, T. Lindahl, C.P. Ponting, F.M. Ashcroft, S.O. Rahilly and C.J. Schofield, Science, 2007, 318, 1469-1472.

14. R.P. Hausinger, Crit. Rev. Biochem. Mol. Biol., 2004, 39, 21-68.

15. G. Jia, Y. Fu, X. Zhao, Q. Dai, G. Zheng, Y. Yang, C. Yi, T. Lindahl, T. Pan, Y.G. Yang and C. He, Nat. Chem. Biol., 2011, 7, 885-887.

16. L. Rohena, M. Lawson, E. Guzman, M. Ganapathi, M.T. Cho, E. Haverfield and K. Anyane Yeboa, Am. J. Med. Genet A., 2016, 170, 1023-1028.

17. H. Daoud, D. Zhang, F. McMurray, A. Yu, S.M. Luco, J. Vanstone, O. Jarinova, N. Caron, 
J. Wickens, S. Shishodia, H. Choi, M.A. McDonough, C.J Schofield, M.E. Harper, D.A. Dyment and C.M. Armour, J. Med. Genet., 2016, 53, 200-207.

18. P.J. Holland, T. Hollis, PLoS One. 2010, 5, e8680.

19. A. Vidaki and M. Kayser, Genome Biol. 2017, 18, 238.

20. D. Fang, R.L. Lord and G.A. Cisneros, J. Phys. Chem. B. 2013, 117, 6410-6420.

21. M.G. Quesne, R. Latifi, L.E. Gonzalez-Ovalle, D. Kumar and S.P. de Visser, Chem. Eur. J. 2014, 20, 435-446.

22. B. Wang, Z. Cao, D.A. Sharon, and S. Shaik, ACS Catal. 2015, 5, 7077-7090.

23. H. Torabifard and G.A. Cisneros, Chem. Sci., 2017, 8, 6230-6238.

24. H. Liu, J. Llano, and J.W. Gauld, J. Phys. Chem. B 2009, 113, 4887-4898.

25. B. Bleijlevens, T. Shivarattan, E. Flashman, Y. Yang, P.J. Slimpson, P. Koivisto, B. Sedgwick, C.J. Schofield and S.J. Mathews, EMBO Rep. 2008, 9, 872-877.

26. R. Chowdhury, I.K.H. Leung, Y. Tian, M.I. Abboud, W. Ge, C. Domene, F. Cantrelle, I. Landrieu, A.P. Hardy, C.W. Pugh, P.J. Ratcliffe, T.D.W. Claridge and C.J. Schofield, Nat. Commun. 2016, 7, 12673.

27. N.R. Rose, M.A. McDonough, O.N.F. King, A. Kawamura and C.J. Schofield, Chem. Soc. Rev. 2011, 40, 4364-4397.

28. C.Z. Christov, A. Lodola, T.G. Karabencheva-Christova, S. Wan, P.V. Coveney, and A.J. Mulholland, Biophys J., 2013, 104, L5-L7.

29. T.G. Karabencheva-Christova, J. Torras, A.J. Mulholland, A. Lodola and C.Z. Christov, Scientific Reports, 2017, 7, 17395.

30. W. Singh, G.B. Fields, C.Z. Christov and T.G. Karabencheva-Christova, RSC Adv., 2016, 6, 23223.

31. J. Ainsley, A.J. Mulholland, G.W. Black, O, Sparagano, C.Z. Christov, and T.G. Karabencheva-Christova, ACS Omega, 2018, 3, 4847-4859.

32. T.G. Karabencheva-Christova, C.Z. Christov and G.B. Fields, J. Phys. Chem. B, 2018, 122, 5316-5326.

33. X. Pang, K. Han and Q. Cui, J. Comput. Chem. 2013, 34, 1620-1635.

34. A. Fiser and A. Sali, Method Enzymol. 2003, 374, 461.491.

35. C.G. Yang, C. Yi, E.M. Duguid, C.T. Sullivan, P.A. Rice and C. He, Nature, 2008, 452, 961965. 
36. J.C. Gordon, J.B. Myers, T. Folta, V. Shoja, L.S. Heath, and A. Onufriev, Nuclei Acids Res., 2005, 33, W368-371.

37. P. Li and K.M. Merz, J. Chem. Info. Model. 2016, 56, 599-604.

38. R. Salomon-Ferrer, A.W. Götz, D. Poole, S. Le Grand, amd R.C. Walker, J. Chem. Theory Comput. 2013, 9, 3878-3888.

39. D. Case, V. Babin, J. Berryman, R. Betz, Q. Cai, D. Cerutti, T. Cheatham III, T. Darden, R. Duke and H. Gohlke, Amber, 2014, 14, 29-31.

40. J.A. Maier, C. Martinez, K. Kasavajhala, L. Wickstrom, K.E: Hauser, and C. Simmerling, J. Chem. Theory Comput 2015, 11, 3696-3713.

41. W.L. Jorgensen, J. Chandrasekhar, J.D. Madura, R.W. Impey, and M.L. Klein, J. Chem. Phys. 1983, 79, 926-935.

42. T. Darden, D, York, and L. Pedersen, J. Chem. Phys. 1993, 98, 10089-10092.

43. R. Davidchack, R. Handel and M.V. Tretyakov, J. Chem. Phys. 2009, 130, 234101.

44. J.P. Ryckaert, G. Ciccotti, and H.J. Berendsen, J. Comput. Phys. 1977, 23, 327-341.

45. H.J. Berendsen, J.V. Postma, W.F. van Gunsteren, A. DiNola, and J. Haak, J. Chem. Phys. 1984, 81, 3684-3690.

46. D.R. Roe and T.E. Cheatham III, J. Chem. Theory Comput. 2013 9, 3084-3095.

47. W. Humphrey, A. Dalke and K. Schulten, J. Mol. Graph. 1996, 14, 27-38.

48. E.F. Pettersen, T.D. Goddard, C.C. Huang, G.S. Couch, D.M. Greenblatt, E.C. Meng, and T.E. Ferrin, J. Comput. Chem. 2004, 25, 1605-1612.

49. B.J. Grant, A.P. Rodrigues, K.M. ElSawy, J.A. McCammon, and L.S. Caves, Bioinformatics (Oxford, England) 2003, 22, 2695-2696.

50. P. Tao and H.B. Schlegel, J. Comput. Chem. 2010, 31, 2363-2369.

51. F. Maseras and K. Morokuma, J. Comput. Chem. 1995, 16, 1170-1179.

52. M. Svensson, S. Humbel, and K. Morokuma, J. Chem. Phys. 1996, 105, 3654-3661.

53. S. Dapprich, I. Komáromi, K.S. Byun, K. Morokuma and M.J. Frisch, J. Mol. Struc: THEOCHEM 1999, 461, 1-21.

54. T. Vreven, K. Morokuma, O. Farkas, H.B. Schlegel, and M.J. Frisch, J. Comput. Chem. 2003, 24, 760-769.

55. T. Vreven and K. Morokuma, J. Comput. Chem. 2000, 21, 1419-1432.

56. M.J.T. Frisch, G. W. Schlegel, H. B. Scuseria, G. E. Robb, M. A. Cheeseman, J. R. Scalmani, 
G. Barone, V. Mennucci, B. Petersson, G. A. Nakatsuji, H. Caricato, M. Li, X. Hratchian, H. P. Izmaylov, A. F. Bloino, J. Zheng, G. Sonnenberg, J. L. Hada, M. Ehara, M. Toyota, K. Fukuda, R. Hasegawa, J. Ishida, M. Nakajima, T. Honda, Y. Kitao, O. Nakai, H. Vreven, T. Montgomery, J. A., Jr. Peralta, J. E. Ogliaro, F. Bearpark, M. Heyd, J. J. Brothers, E. Kudin, K. N. Staroverov, V. N. Kobayashi, R. Normand, J. Raghavachari, K. Rendell, A. Burant, J. C. Iyengar, S. S. Tomasi, J. Cossi, M. Rega, N. Millam, N. J. Klene, M. Knox, J. E. Cross, J. B. Bakken, V. Adamo, C. Jaramillo, J. Gomperts, R. Stratmann, R. E. Yazyev, O. Austin, A. J. Cammi, R. Pomelli, C. Ochterski, J. W. Martin, R. L. Morokuma, K. Zakrzewski, V. G. Voth, G. A. Salvador, P. Dannenberg, J. J. Dapprich, S. Daniels, A. D. Farkas, Ö. Foresman, J. B. Ortiz, J. V. Cioslowski, J. Fox, Gaussian 09. Gaussian, Inc Revision D.01(Wallingford CT) 2009.

57. H.M. Senn and W. Thiel, Angew. Chem. Int. Ed. 2009, 48, 1198-1229.

58. P.A. Kollman, I. Massova, C. Reyes, B. Kuhn, S. Huo, L. Chong, M. Lee, T. Lee, Y. Duan, W. Wang, O. Donini, P. Cieplak, J. Srinivasan, D.A. Case, and T.E. 3rd Cheatham, Acc. Chem. Res. 2000, 33, 889-897.

59. I. Massova and P.A. Kollman, Perspectives in Drug Discovery and Design 2000, 18, 113 135.

60. V. Tsui and D.A. Case, Biopolymers 2000, 56, 275-291.

61. M. Padariya and U. Kalathiya, Comp. Biol. Chem. 2016, 64, 414-425.

62. T.A. Muller and R.P. Hausinger in 2-Oxoglutarate-Dependent Oxygenases, ed. C.J. Schofield and R.P. Hausinger, Royal Society of Chemistry, Cambridge, 2015, series No. 3, ch. 8, pp. 246-262.

63. M. Mantri, Z. Zhang, M.A. McDonough, O.N.F. King, A. Kawamura and C.J. Schofield, FEB Journal, 2012, 279, 1563-1575.

64. T.L. Yeh, T.M. Leissing, M.I. Abboud, C.C. Thinnes, O. Atasoylu, J.P. Holt-Martyn, D. Zhang, A. Tumber, K. Lippi, C.T. Lohans, I.K.H. Leung, H. Morcrette, I.J. Clifton, T.D.W. Claridge, A. Kawamura, E. Flashman, X. Lu, P.J. Ratcliffe, R. Chowdhury, C.W. Pugh and C.J. Schofield, Chem. Sci. 2017, 8, 7651-7668. 